\title{
OPEN Interrelationship
}

\section{between 2019-nCov receptor DPP4 and diabetes mellitus targets based on protein interaction network}

\author{
Qian Gao ${ }^{凶}$, Wenjun Zhang, Tingting Li, Guojun Yang, Wei Zhu, Naijun Chen \& Huawei Jin
}

Patients with diabetes are more likely to be infected with Coronavirus disease 2019 (COVID-19), and the risk of death is significantly higher than ordinary patients. Dipeptidyl peptidase-4 (DPP4) is one of the functional receptor of human coronavirus. Exploring the relationship between diabetes mellitus targets and DPP4 is particularly important for the management of patients with diabetes and COVID19. We intend to study the protein interaction through the protein interaction network in order to find a new clue for the management of patients with diabetes with COVID-19. Diabetes mellitus targets were obtained from GeneCards database. Targets with a relevance score exceeding 20 were included, and DPP4 protein was added manually. The initial protein interaction network was obtained through String. The targets directly related to DPP4 were selected as the final analysis targets. Importing them into String again to obtain the protein interaction network. Module identification, gene ontology (GO) analysis and Kyoto encyclopedia of genes and genomes (KEGG) pathway analysis were carried out respectively. The impact of DPP4 on the whole network was analyzed by scoring the module where it located. 43 DPP4-related proteins were finally selected from the diabetes mellitus targets and three functional modules were found by the cluster analysis. Module 1 was involved in insulin secretion and glucagon signaling pathway, module 2 and module 3 were involved in signaling receptor binding. The scoring results showed that LEP and apoB in module 1 were the highest, and the scores of INS, IL6 and ALB of cross module associated proteins of module 1 were the highest. DPP4 is widely associated with key proteins in diabetes mellitus. COVID-19 may affect DPP4 in patients with diabetes mellitus, leading to high mortality of diabetes mellitus combined with COVID-19. DPP4 inhibitors and IL-6 antagonists can be considered to reduce the effect of COVID-19 infection on patients with diabetes.

Due to the high prevalence and long incubation periods often without symptoms, the severe acute respiratory syndrome coronavirus-2 (SARS-CoV-2) has infected hundreds of millions of people around the world, causing the coronavirus disease 2019 (COVID-19) pandemic ${ }^{1}$. Recent studies have shown that dipeptidyl peptidase4 (DPP4) is one of the functional receptor of human coronavirus ${ }^{2-5}$. SARS-CoV-2 Virus S protein can infect the body by acting on this receptor of bronchiolar epithelial cells ${ }^{6}$. DPP4 also known as CD26, is a serine exopeptidase, a multifunctional type-II transmembrane glycoprotein that presents in a dimeric form on the cell surface. DPP4 is multifunctional, highly conserved among mammals. It regulates the activities of peptide hormones, neuropeptides, cytokines and growth factors, but also act as a surface antigen to cooperate with other molecules or proteins to mediate the interaction between cells and matrix, cells and cells, and play various regulatory roles in immune activation, inflammatory response and tumorigenesis ${ }^{7}$.

DPP4 inhibitors are a kind of hypoglycemic drugs which have been widely used in patients with diabetes. DPP4 can decompose glucagon like peptide-1 (GLP-1), which can stimulate insulin secretion. DPP4 inhibitors can effectively antagonize this effect and then control blood glucose, especially postprandial blood glucose, and improve glucose tolerance, insulin resistance and other symptoms of patients by inhibiting the degradation of GLP- $1^{8}$. Patients with diabetes are more likely to be infected with COVID-19, and the risk of death is significantly higher than ordinary patients ${ }^{9,10}$. Current studies have shown that DPP4 inhibitors can be considered as the preferred hypoglycemic regimen in the treatment of patients with diabetes with COVID-19 infection ${ }^{11}$. Exploring the relationship between DPP4 and diabetes targets is particularly important for the management of patients 
with diabetes and COVID-19.In order to strengthen the management of patients with diabetes with COVID19 , we intend to study the protein interaction through the protein interaction network, and evaluate the degree of protein-protein correlation through the correlation score. Based on the String database, this paper analyzes the relationship between DPP4 and diabetic protein targets, in order to find a new clue for the management of patients with diabetes with COVID-19.

\section{Materials and methods}

Get diabetes mellitus targets information. Taking 'diabetes mellitus' as the keyword, diabetes mellitus-related targets were searched in GeneCards database (https://www.genecards.org/). The target with a relevance score exceeding 20 was selected as the research target.

Screening of protein targets directly associated with DPP4. The diabetes mellitus related target (obtained by step 2.1) and DPP4 were imported into STRING database (https://string-db.org/) ${ }^{12}$ in the form of symbol to obtain the protein-protein interaction network map. And then the targets directly related to DPP4 were screened for further research.

Construction of DPP4 related protein network. DPP4 and 43 related proteins were re-imported into STRING to obtain the protein-protein interaction network for subsequent analysis.

Identification and analysis of DPP4 related protein network modules. In the STRING website, $\mathrm{K}$-means algorithm was used to cluster the DPP4 and 43 related proteins.

Gene ontology (GO) and Kyoto encyclopedia of genes and genomes (KEGG) pathway analysis of each module. The enrichment analysis of GO function and KEGG pathway analysis was carried out through STRING to predict its action mechanism and construct the network diagram.

Evaluation of the impact of DPP4 on the whole protein network. In order to further evaluate the impact of DPP4 on the whole network and the possible pathway, the module 1 where DPP4 is located is used as the internal network, the cross module association of internal network is screened out. The statistical description and mapping are carried out. The data are filtered with 0.6 (determined by mean and median) as the standard, the qualified cross module associated proteins and associated scores were listed, and the proteins included in module 1 and the cross module associated protein scores of module 1 were summed as the scores.

\section{Results}

Screening results of DPP4 associated proteins. A total of 1031 diabetes mellitus targets and 43 targets directly related to DPP4 were obtained by screening (Table 1).

Protein interaction network results. The network consists of 44 nodes and 570 edges. The average value of nodes is 25.9 (Fig. 1).

Cluster analysis results of protein interaction network. Three functional modules were obtained by cluster analysis. Module 1 includes 17 nodes (SLC5A2, SLC2A2, LEP, GCG, DPP4, ADIPOQ, APOB, GGT1, GPT, NPY, GCK, CPE, SST, GCGR, IAPP, GLP1R, GHRL); module 2 includes 14 nodes (ICAM1, VCAM1, APOE, CCL2, TNF, CRP, IL6, ALB, ACE, HNF1A, CCR5, CXCL10, IL10, PPAARG); module 3 includes 13 nodes (MMP2, MMP9, FN1, VEGFA, CAV1, CTLA4, NOS3, AKT1, INS, SLC2A4, REN, SERPINE1, INS-IGF2) (Fig. 2).

GO analysis and KEGG analysis. Through GO analysis and KEGG analysis, it was found that the target of module 1 was mainly enriched in insulin secretion and glucagon signal transduction pathway; the target of module 2 and module 3 was mainly enriched in signaling receptor binding.

Module 1 cross module correlation statistics results. Module 1 contains 190 cross module associations, accounting for $33 \%$ of the total number of network associations (570). The average score of association is 0.649 , and the median is 0.628 (Table 2).

Module 1 cross module correlation impact assessment results. In module 1, LEP and apoB have the highest scores, which indicate that the above proteins may have cross module effects. The scores of INS, IL6 and ALB of cross module associated proteins of module 1 were the highest, which indicates that the effects of module 1 on other modules are more likely to be achieved through interaction with the above proteins (Table 3).

\section{Discussion}

By sorting the correlation scores of DPP4 related diabetes targets, we found that GCG, INS and GLP1R had the highest correlation scores, which were more than 0.8 , and GCG and INS were more than 0.9 , which meant that DPP4 was most closely related to the above diabetes targets. By cluster analysis, three functional modules were found. Module 1 which contain DPP4 was mainly involved in insulin secretion and glucagon signal transduction pathway, while module 2 and module 3 were involved in signaling receptor binding. The binding of $S$ protein 


\begin{tabular}{|c|c|c|}
\hline Symbol & Protein name & Score \\
\hline ACE & Angiotensin-converting enzyme & 0.709 \\
\hline ADIPOQ & Adiponectin & 0.572 \\
\hline AKT1 & RAC-alpha serine/threonine-protein kinase & 0.52 \\
\hline ALB & Serum albumin & 0.671 \\
\hline APOB & Apolipoprotein B-100 & 0.438 \\
\hline APOE & Apolipoprotein E & 0.422 \\
\hline CAV1 & Caveolin-2 & 0.692 \\
\hline CCL2 & $\mathrm{C}-\mathrm{C}$ motif chemokine 2 & 0.448 \\
\hline CCR5 & C-C chemokine receptor type 5 & 0.4 \\
\hline $\mathrm{CPE}$ & Carboxypeptidase E & 0.438 \\
\hline CRP & C-reactive protein & 0.536 \\
\hline CTLA4 & Cytotoxic T-lymphocyte protein 4 & 0.44 \\
\hline CXCL10 & C-X-C motif chemokine 10 & 0.581 \\
\hline FN1 & Fibronectin type III domain containing & 0.773 \\
\hline GCG & Glucagon & 0.994 \\
\hline GCGR & Glucagon receptor & 0.573 \\
\hline GCK & Glucokinase & 0.461 \\
\hline GGT1 & Glutathione hydrolase 1 proenzyme & 0.411 \\
\hline GHRL & Appetite-regulating hormone & 0.559 \\
\hline GLP1R & Glucagon-like peptide 1 receptor & 0.899 \\
\hline GPT & Alanine aminotransferase 1 & 0.508 \\
\hline HNF1A & Hepatocyte nuclear factor 1-alpha & 0.538 \\
\hline IAPP & Islet amyloid polypeptide & 0.672 \\
\hline ICAM1 & Intercellular adhesion molecule 1 & 0.441 \\
\hline IL10 & Interleukin-10 & 0.41 \\
\hline IL6 & Interleukin-6 & 0.514 \\
\hline INS & Insulin & 0.942 \\
\hline INS-IGF2 & Insulin, isoform 2 & 0.535 \\
\hline LEP & Leptin & 0.593 \\
\hline MMP2 & $72 \mathrm{kDa}$ type IV collagenase & 0.404 \\
\hline MMP9 & Matrix metalloproteinase- 9 & 0.405 \\
\hline NOS3 & Nitric oxide synthase, endothelial & 0.658 \\
\hline NPY & Pro-neuropeptide $\mathrm{Y}$ & 0.735 \\
\hline PPARG & Peroxisome proliferator-activated receptor gamma & 0.51 \\
\hline REN & Renin & 0.499 \\
\hline SERPINE1 & Plasminogen activator inhibitor 1 & 0.454 \\
\hline SLC2A2 & Solute carrier family 2 , facilitated glucose transporter member 2 & 0.462 \\
\hline SLC2A4 & Solute carrier family 2 , facilitated glucose transporter member 4 & 0.461 \\
\hline SLC5A2 & Sodium/glucose cotransporter 2 & 0.892 \\
\hline SST & Somatostatin & 0.472 \\
\hline TNF & Tumor necrosis factor & 0.473 \\
\hline VCAM1 & Vascular cell adhesion protein 1 & 0.416 \\
\hline VEGFA & Vascular endothelial growth factor A & 0.643 \\
\hline
\end{tabular}

Table 1. DPP4 related protein.

of COVID-19 with DPP4 was the starting point of COVID-19. In order to evaluate the possible pathogenesis of the virus after entering the human body, DPP4 was taken as the starting point of the whole network for in-depth analysis. Because there were a lot of low values in the correlation scores of DPP 4 cross module associated proteins, the mean and median were used as the standard to filter the data, and the sum of the correlation scores was used as the score. It was found that the scores of LEP and apoB were the highest in module 1. The scores of INS, IL6 and ALB of cross module associated proteins of module 1 were the highest. It is worth noting that DPP 4 cross module correlation scores are less than 0.6 , which do not been shown. Therefore, the abnormal changes of DPP4 may not play an effect by directly interacting with module associated proteins, but may be that module 1 magnifies its effect, and this effect is transmitted to module 2 and module 3 through the close relationship between module 1 and INS, IL6 and ALB, resulting in the disorder of glucose metabolism and inflammatory regulation. 


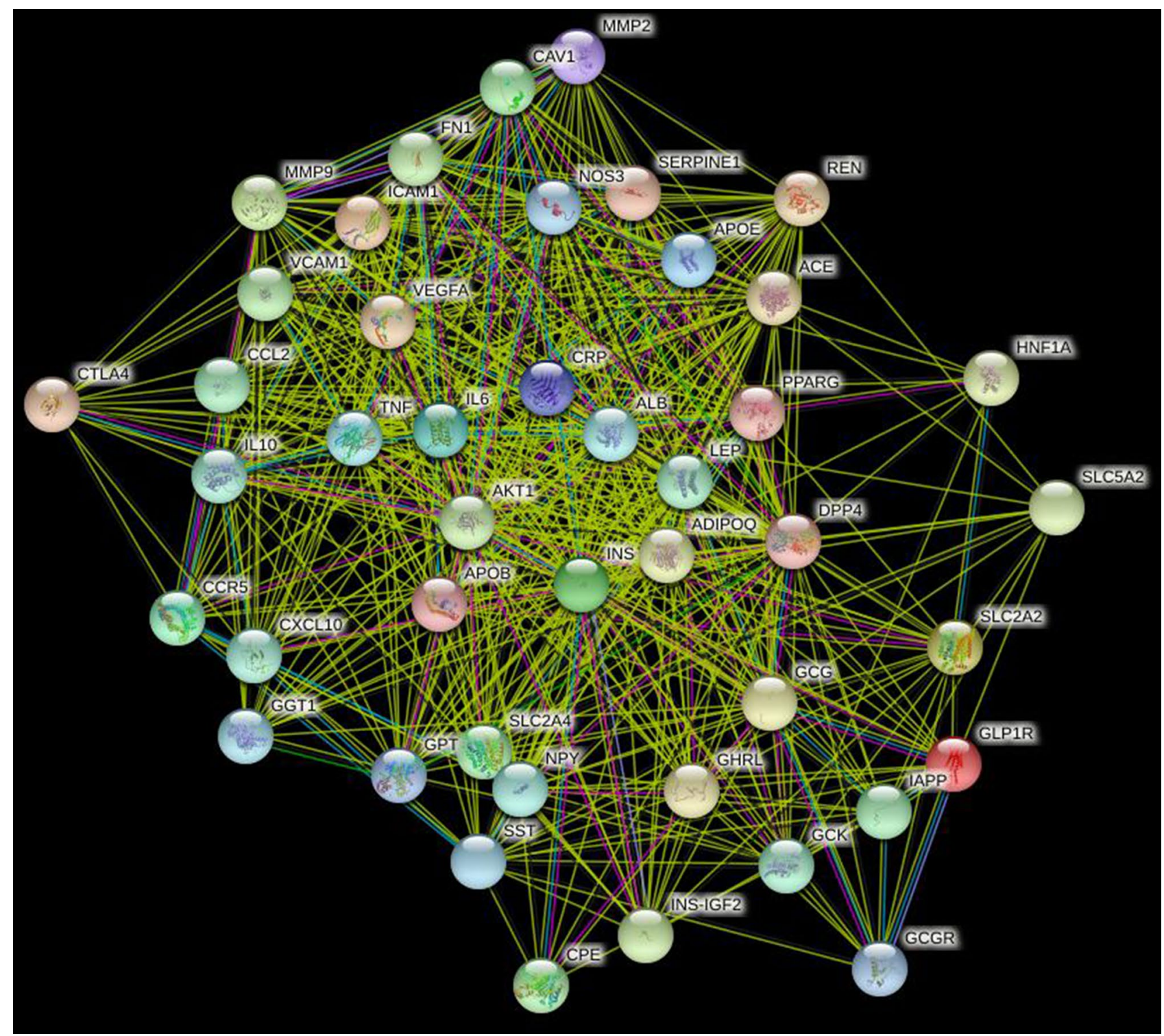

Figure 1. Interaction networ of DPP4 related protein.

Clinical trials show that patients with diabetes were more likely to be infected with SAR-COV-2, while the prognosis of patients with diabetes was worse and the risk of death was higher ${ }^{9,10}$. So, what was the mechanism of susceptibility to SAR-COV-2 in patients with diabetes? What was the mechanism of higher risk of death and worse prognosis in patients with diabetes? How to guide the medication of patients with diabetes infected with SAR-COV-2 in clinic? DPP4 as a type II transmembrane protein was also known to be cleaved from the cell membrane involving different metalloproteases in a cell-type-specific manner. Circulating, soluble DPP4 had been identified as a adipokine, which exerts both para- and endocrine effects ${ }^{13}$. Recently, studies found that sCD26 serum protein levels are reduced in diabetes. High serum sCD26 level could protect from viral infection by blocking the receptor from virus entry, whereas low sCD26 level may be associated with a higher risk of infection which may be one of the mechanisms of susceptibility to SARS-COV-2 in patients with diabetes ${ }^{14}$. The high mortality of patients with COVID-19 was closely related to the disorder of glucose metabolism and inflammation regulation ${ }^{15}$. The S protein of SARS-COV-2 can invade T, NK and other immune cells through binding receptor DPP4, and activate nuclear factor $-\kappa \mathrm{B}(\mathrm{NF}-\kappa \mathrm{b})$ pathway, resulting in the secretion of a series of pro-inflammatory cytokines, including IL- ${ }^{16}$. IL-6 can promote the differentiation of T helper cell 17 (Th17) and other lymphocyte changes. Circulating IL-6 and soluble IL-6 receptor complexes indirectly activate many types of cells, including endothelial cells, leading to the proliferation of a series of cytokines, leading to decreased blood pressure and acute respiratory distress syndrome (ARDS) ${ }^{17}$. IL-6 plays a key role in this cascade. It suggest the possibility of IL-6 antagonists (such as tocilizumab, sarilumab and siluximab) been used in severe COVID-19 disease. DPP4 also degraded GLP-1 and Gastric Inhibitory Polypeptide (GIP) and played an important role in glucose metabolism. Studies shown that GLP-1-based therapy can reduce the activation of immune cells, inhibit the release of pro-inflammatory cytokines, and reduce organ dysfunction and mortality ${ }^{18}$. DPP4 


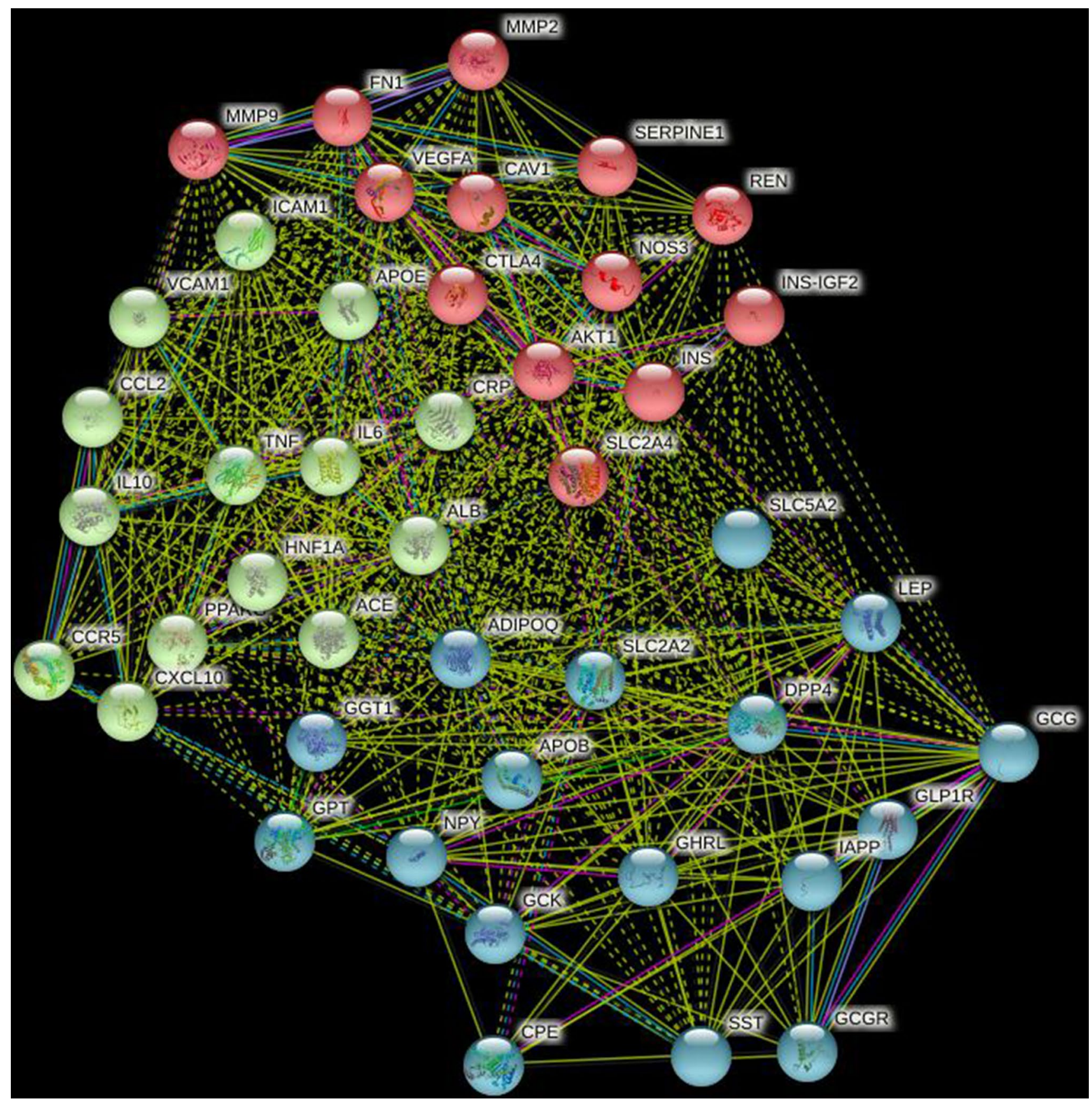

Figure 2. DPP4 related protein module recognition.

inhibitor increased the half-life of GLP-1 and therefore prolonged the half-life of insulin. Because of this, DPP4 inhibitor became a major target for the treatment of patients with diabetes. DPP4 inhibitors can also resist lung inflammation and reduce lung injury ${ }^{19}$. These studies suggested that DPP4 inhibitors may play an active role in the treatment of patients with diabetes with COVID-19. However, the issue remained controversial. Males believed that DPP4 inhibitors can inhibit the immune system and may increase the risk of infection ${ }^{20}$. However, there were also studies show that DPP4 inhibitors do not have a negative impact ${ }^{21}$. Previous retrospective studies found that DPP4 inhibitors have serious heterogeneity in the treatment effect of COVID-1922-28. However, these studies were not randomized controlled double-blind studies. At present, three randomized controlled trials (NCT 04341935, NCT 04371978 and NCT04365517) (Retrieved from: https://clinicaltrials.gov/) were ongoing to study the effect of DPP4 inhibitors on the prognosis of COVID-19 and the clinical results were expected to be obtained as soon as possible.

Leptin which was a hormone secreted by adipose tissue had the highest cross module effect in module 1, with a score of 16.071. When the body fat was reduced or in a low-energy state (such as starvation), leptin decreased significantly, thus stimulating the food seeking behavior and reducing its own energy consumption. On the contrary, when the body fat increased, leptin increased, which inhibited eating and accelerates metabolism ${ }^{29}$. 


\begin{tabular}{|c|c|c|c|}
\hline Module & Protein & GO and KEGG analysis & $P$ \\
\hline Module 1 & $\begin{array}{l}\text { SLC5A2, SLC2A2, LEP, GCG, DPP4, ADIPOQ, APOB, GGT1, GPT, NPY, GCK, CPE, SST, GCGR, IAPP, } \\
\text { GLP1R, GHRL }\end{array}$ & $\begin{array}{l}\text { GO: } \\
\text { hormone activity } \\
\text { KEGG: } \\
\text { Maturity onset diabetes of the young; } \\
\text { Insulin secretion; } \\
\text { Glucagon signaling pathway }\end{array}$ & $\begin{array}{l}1.29 \mathrm{e}-07 \\
3.55 \mathrm{e}-05 \\
3.55 \mathrm{e}-053.55 \mathrm{e}-05\end{array}$ \\
\hline Module 2 & $\begin{array}{l}\text { ICAM1, VCAM1, APOE, CCL2, TNF, CRP, IL6, ALB, ACE, HNF1A, CCR5, CXCL10, IL10, CCL2, } \\
\text { PPAARG }\end{array}$ & $\begin{array}{l}\text { GO: } \\
\text { signaling receptor binding } \\
\text { KEGG: } \\
\text { African trypanosomiasis; } \\
\text { TNF signaling pathway }\end{array}$ & $\begin{array}{l}2.63 \mathrm{e}-05 \\
9.61 \mathrm{e}-08 \\
9.61 \mathrm{e}-08\end{array}$ \\
\hline Module 3 & MMP2, MMP9, FN1, VEGFA, CAV1, CTLA4, NOS3, AKT1, INS, SLC2A4, REN, SERPINE1, INS-IGF2 & $\begin{array}{l}\text { GO: } \\
\text { signaling receptor binding } \\
\text { KEGG: } \\
\text { Fluid shear stress and atherosclerosis }\end{array}$ & $\begin{array}{l}0.0030 \\
2.14 \mathrm{e}-08\end{array}$ \\
\hline
\end{tabular}

Table 2. GO and KEGG analysis of different modules.

\begin{tabular}{|c|c|c|c|c|c|c|c|c|c|}
\hline \multicolumn{2}{|l|}{ APOB } & \multicolumn{2}{|c|}{ GGT1 } & \multicolumn{2}{|l|}{ GPT } & \multicolumn{2}{|l|}{ NPY } & \multicolumn{2}{|l|}{ CPE } \\
\hline NOS3 & 0.707 & IL6 & 0.616 & IL10 & 0.66 & IL6 & \begin{tabular}{|l|}
0.709 \\
\end{tabular} & INS & 0.97 \\
\hline VCAM1 & 0.74 & CRP & 0.669 & TNF & 0.707 & INS & 0.889 & & \\
\hline IL10 & 0.751 & INS & 0.67 & IL6 & 0.733 & CXCL10 & 0.908 & & \\
\hline MMP9 & 0.789 & ALB & 0.805 & INS & 0.839 & CCR5 & 0.919 & & \\
\hline TNF & 0.793 & & & CRP & 0.896 & & & & \\
\hline INS & 0.848 & & & ALB & \begin{tabular}{|l|}
0.924 \\
\end{tabular} & & & & \\
\hline IL6 & 0.969 & & & & & & & & \\
\hline \multicolumn{2}{|l|}{ SST } & \multicolumn{2}{|c|}{ GCGR } & \multicolumn{2}{|l|}{ GHRL } & \multicolumn{2}{|l|}{ GCG } & \multicolumn{2}{|l|}{ LEP } \\
\hline ALB & 0.621 & INS & 0.694 & INS-IGF2 & 0.607 & INS-IGF2 & 0.668 & REN & 0.662 \\
\hline AKT1 & 0.68 & & & NOS3 & 0.628 & SERPINE1 & 0.672 & CAV1 & 0.682 \\
\hline CXCL10 & 0.908 & & & IL6 & 0.63 & ALB & 0.704 & INS-IGF2 & 0.7 \\
\hline INS & 0.915 & & & ALB & 0.675 & AKT1 & 0.776 & MMP9 & 0.707 \\
\hline \multirow[t]{16}{*}{ CCR5 } & 0.922 & & & AKT1 & 0.71 & SLC2A4 & 0.793 & VCAM1 & 0.722 \\
\hline & & & & INS & 0.944 & INS & 0.986 & MMP2 & 0.724 \\
\hline & & & & & & & & CCL2 & 0.732 \\
\hline & & & & & & & & ICAM1 & 0.762 \\
\hline & & & & & & & & NOS3 & 0.762 \\
\hline & & & & & & & & APOE & 0.775 \\
\hline & & & & & & & & IL10 & 0.797 \\
\hline & & & & & & & & ALB & 0.803 \\
\hline & & & & & & & & AKT1 & 0.841 \\
\hline & & & & & & & & SLC2A4 & 0.856 \\
\hline & & & & & & & & VEGFA & 0.867 \\
\hline & & & & & & & & SERPINE1 & 0.879 \\
\hline & & & & & & & & TNF & 0.9 \\
\hline & & & & & & & & IL6 & 0.943 \\
\hline & & & & & & & & INS & 0.976 \\
\hline & & & & & & & & CRP & 0.981 \\
\hline
\end{tabular}

Table 3. Module 1 cross module associated protein and relevance score.

Leptin was also associated with monocyte activation and severe illness in patients with COVID-1930. Overweight patients with COVID-19 tended to have higher leptin levels, which further activated monocytes, leading to amplification or imbalance of immune response, which may also be the mechanism of overweight patients more prone to serious diseases ${ }^{31}$. Cytokine release syndrome was an important cause of death in patients with COVID-19. However, the changes of cytokine profile and the underlying mechanism were still unknown. Using the cytokine array containing 174 cytokines related to inflammation found that the cytokine spectrum of severe patients with COVID-19 was significantly different from that of mild patients or healthy controls. Leptin, CXCL-10, IL-6, IL-10, IL-12, TNF and other cytokines, indicating that these inflammatory factors can predict the severity of COVID -19 disease ${ }^{32}$. The cluster analysis showed that leptin in module 1 and IL-6, IL-10, TNF in module 2 had a cross model effect, and the highest score of IL-6 is 6.163, which further supported the possibility of IL-6 antagonists in the treatment of severe COVID-19 disease. 
In this paper, we screened out the diabetes targets and functional modules closely related to DPP4 through protein interaction network, and analyzed the influence of DPP4 module 1 on the whole protein network and the possible pathway. It was proposed that the influence of COVID-19 infection was amplified by DPP4 in patients with diabetes, and through its interaction with INS, leptin, IL-6 and other proteins. Increased glucose metabolism disorder and excessive inflammatory reaction lead to the high mortality in patients with diabetes with COVID-19. At present, the data of retrospective observational studies showed that the therapeutic effect of DPP4 inhibitors had serious heterogeneity, but the strength of the above studies was low. We look forward to further randomized controlled trials to verify our inference.

\section{Conclusions}

DPP4 was widely associated with key proteins in diabetes mellitus. COVID-19 may affect DPP4 in patients with diabetes mellitus, leading to high mortality of diabetes mellitus combined with COVID-19. DPP4 inhibitors and IL- 6 antagonists can be considered to reduce the effect of COVID-19 infection on patients with diabetes.

Received: 23 June 2021; Accepted: 10 December 2021

Published online: 07 January 2022

\section{References}

1. Chung, J. Y., Thone, M. N. \& Kwon, Y. J. COVID-19 vaccines: The status and perspectives in delivery points of view. Adv. Drug Deliv. Rev. 170, 1-25 (2020).

2. Brooke, G. N. \& Prischi, F. Structural and functional modelling of SARS-CoV-2 entry in animal models. Sci. Rep. 10(1), 15917 (2020).

3. Vankadari, N. \& Wilce, J. A. Emerging WuHan (COVID-19) coronavirus: Glycan shield and structure prediction of spike glycoprotein and its interaction with human CD26. Emerg. Microbes Infect. 9(1), 601-604 (2020).

4. Li, Y., Zhang, Z., Yang, L., et al. The MERS-CoV receptor DPP4 as a candidate binding target of the SARS-CoV-2 spike. iScience 23(6):101160 (2020).

5. Radzikowska, U., Ding, M., Tan, G, et al. Distribution of ACE2, CD147, CD26, and other SARS-CoV-2 associated molecules in tissues and immune cells in health and in asthma, COPD, obesity, hypertension, and COVID-19 risk factors. Allergy (2020).

6. K KDD, S SKK, R RTT, et al. Coronavirus disease 2019-COVID-19. Clin. Microbiol. Rev. 33(4) (2020).

7. Raj, V. S. et al. Dipeptidyl peptidase 4 is a functional receptor for the emerging human coronavirus-EMC. Nature 495(7440), 251-254 (2013).

8. Saito, T. et al. Polyarthropathy in type 2 diabetes patients treated with DPP4 inhibitors. Diabetes Res. Clin. Pract. 102(1), e8-e12 (2013).

9. Fang, L., Karakiulakis, G. \& Roth, M. Are patients with hypertension and diabetes mellitus at increased risk for COVID-19 infection?. Lancet Respir. Med. 8(4), e21 (2020).

10. Yang, X. et al. Clinical course and outcomes of critically ill patients with SARS-CoV-2 pneumonia in Wuhan, China: A singlecentered, retrospective, observational study. Lancet Respir. Med. 8(5), 475-481 (2020).

11. Solerte, S. B., Di Sabatino, A., Galli, M. \& Fiorina, P. Dipeptidyl peptidase-4 (DPP4) inhibition in COVID-19. Acta Diabetol. 57(7), 779-783 (2020).

12. Von Mering, C. et al. STRING: A database of predicted functional associations between proteins. Nucl. Acids Res. 31, 258-261 (2003).

13. Röhrborn, D., Wronkowitz, N. \& Eckel, J. DPP4 in diabetes. Front. Immunol. 6, 386 (2015).

14. Raha, A. A., Chakraborty, S., Henderson, J., et al. Investigation of CD26, a potential SARS-CoV-2 receptor, as a biomarker of age and pathology. Biosci. Rep. 40(12) (2020).

15. Ye, Q., Wang, B. \& Mao, J. The pathogenesis and treatment of the 'Cytokine Storm' in COVID-19. J. Infect. 80(6), 607-613 (2020).

16. Wang, J. \& Meng, W. COVID-19 and diabetes: The contributions of hyperglycemia. J. Mol. Cell Biol. 10(1), mjaa054 (2020).

17. McGonagle, D. et al. The role of cytokines including interleukin-6 in COVID-19 induced pneumonia and macrophage activation syndrome-like disease. Autoimmun. Rev. 19(6), 102537 (2020).

18. Shah, F. A. et al. Therapeutic effects of endogenous incretin hormones and exogenous incretin-based medications in sepsis. J. Clin. Endocrinol. Metab. 104(11), 5274-5284 (2019).

19. Jang, J. H. et al. Anti-inflammatory effects on ischemia/reperfusion-injured lung transplants by the cluster of differentiation $26 /$ dipeptidylpeptidase 4 (CD26/DPP4) inhibitor vildagliptin. J. Thorac. Cardiovasc. Surg. 153(3), 713-24.e714 (2017).

20. Kokic, M. V. Letter to the editor in response to the article “COVID-19 and diabetes: Can DPP4 inhibition play a role?". Diabetes Res. Clin. Pract. 163, 108163 (2020).

21. Scheen, A. J., Marre, M. \& Thivolet, C. Prognostic factors in patients with diabetes hospitalized for COVID-19: Findings from the CORONADO study and other recent reports. Diabetes Metab. 46(4), 265-271 (2020).

22. Chen, Y. et al. Clinical characteristics and outcomes of patients with diabetes and COVID-19 in association with glucose-lowering medication. Diabetes Care 43(7), 1399-1407 (2020).

23. Cariou, B. \& Hadjadj, S. Phenotypic characteristics and prognosis of inpatients with COVID-19 and diabetes: The CORONADO study. Diabetologia 63(8), 1500-1515 (2020).

24. Fadini, G. P. et al. Exposure to dipeptidyl-peptidase-4 inhibitors and COVID-19 among people with type 2 diabetes: A case-control study. Diabetes Obes. Metab. 22(10), 1946-1950 (2020).

25. Kim, M. K. \& Jeon, J. H. The clinical characteristics and outcomes of patients with moderate-to-severe coronavirus disease 2019 infection and diabetes in Daegu, South Korea. Diabetes Metab. J. 44(4), 602-613 (2020).

26. Mirani, M. \& Favacchio, G. Impact of comorbidities and glycemia at admission and dipeptidyl peptidase 4 inhibitors in patients with type 2 diabetes with COVID-19: A case series from an Academic Hospital in Lombardy, Italy. Diabetes Care 43(12), 3042-3049 (2020).

27. Montastruc, F. et al. Pharmacological characteristics of patients infected with SARS-Cov-2 admitted to Intensive Care Unit in South of France. Therapie 75(4), 381-384 (2020).

28. Solerte, S. B. et al. Sitagliptin treatment at the time of hospitalization was associated with reduced mortality in patients with type 2 diabetes and COVID-19: A multicenter, case-control, retrospective, observational study. Diabetes Care 43(12), 2999-3006 (2020).

29. Zhang, Y. \& Chua, S. Jr. Leptin function and regulation. Compr. Physiol. 8(1), 351-369 (2017).

30. Wang, J. et al. Leptin correlates with monocytes activation and severe condition in COVID-19 patients. J. Leukoc. Biol. 1(6), 1-12 (2021).

31. Alberca RW, Oliveira LM, Branco A, et al. Obesity as a risk factor for COVID-19: an overview. Crit. Rev. Food Sci. Nutr. pp. 1-15 (2020). 
32. McElvaney, O. J. et al. Characterization of the inflammatory response to severe COVID-19 illness. Am. J. Respir. Crit. Care Med. 202(6), 812-821 (2020).

\section{Acknowledgements}

This study was supported by grants from Medical and health projects in Zhejiang Province (Grant NO. 2018KY838).

\section{Author contributions}

Conceptualization and design: Q.G. and W.Z. Data curation: Q.G., N.C., W.Z., G.Y., H.J. Formal analysis: T.L. Writing: Q.G. All authors read and approved the final manuscript.

\section{Competing interests}

The authors declare no competing interests.

\section{Additional information}

Correspondence and requests for materials should be addressed to Q.G.

Reprints and permissions information is available at www.nature.com/reprints.

Publisher's note Springer Nature remains neutral with regard to jurisdictional claims in published maps and institutional affiliations.

(c) Open Access This article is licensed under a Creative Commons Attribution 4.0 International License, which permits use, sharing, adaptation, distribution and reproduction in any medium or format, as long as you give appropriate credit to the original author(s) and the source, provide a link to the Creative Commons licence, and indicate if changes were made. The images or other third party material in this article are included in the article's Creative Commons licence, unless indicated otherwise in a credit line to the material. If material is not included in the article's Creative Commons licence and your intended use is not permitted by statutory regulation or exceeds the permitted use, you will need to obtain permission directly from the copyright holder. To view a copy of this licence, visit http://creativecommons.org/licenses/by/4.0/.

(C) The Author(s) 2022 